\title{
ON THE EXISTENCE OF STEADY GAS FLOW IN PLANE ISOTHERMAL STREAMLINE PATTERNS
}

\author{
R. C. PRIM
}

In this paper we shall investigate the possibility of steady, plane gas flows having streamlines which can be mapped conformally onto a family of parallel lines. We shall limit our consideration to flows of an ideal gas (that is, a nonviscous, thermally nonconducting gas with constant specific heats) in the absence of body forces. The investigation will include rotational as well as irrotational flows.

We shall employ the formulation of the basic gas flow equations due to Munk and Prim [1]. ${ }^{1}$ This formulation includes the most general steady flows of an ideal gas in the absence of body forces.

$$
\operatorname{div}\left[\left(1-w^{2}\right)^{1 /(\gamma-1)} \bar{w}\right]=0,
$$

is the continuity equation and the dynamic equation is

$$
(\bar{w} \cdot \operatorname{grad}) \bar{w}+\frac{\gamma-1}{2 \gamma}\left(1-w^{2}\right) \operatorname{grad} \ln p=0
$$

whence the integrability condition

$$
\operatorname{curl}\left[\frac{\bar{w} \times \operatorname{curl} \bar{w}}{1-w^{2}}\right]=0,
$$

where $\gamma$ denotes the adiabatic exponent, $p$ the pressure, and $\bar{w}$ the reduced velocity vector related to the actual velocity vector $\bar{v}$ by

$$
\bar{v}=\frac{\bar{v}}{v_{\text {ultimate }}}=\frac{\bar{v}}{(2 \gamma /(\gamma-1))(p / \rho)+v^{2}}
$$

where $\rho$ denotes the density.

Equations (1) and (2) will be referred to a plane, isothermal net $\xi, \eta$ in which the squared element of arc length is given by

$$
(d s)^{2}=g(\xi, \eta)\left[(d \xi)^{2}+(d \eta)^{2}\right]
$$

where limitation of the net to a plane requires

$$
\frac{\partial^{2} \ln g}{\partial \xi^{2}}+\frac{\partial^{2} \ln g}{\partial \eta^{2}}=0 .
$$

Presented to the Society, December 29, 1947; received by the editors November 29, 1947.

${ }^{1}$ Numbers in brackets refer to the references cited at the end of the paper. 
The curves $\xi=$ const. will be taken as streamlines. Thus specialized, (1) and (2) become [2]

$$
\frac{\partial}{\partial \eta}\left[g w\left(1-w^{2}\right)^{1 /(\gamma-1)}\right]=0
$$

and

$$
\frac{\partial}{\partial \eta}\left[\frac{w^{2}}{1-w^{2}} \frac{\partial}{\partial \xi} \ln g w\right]=0,
$$

or

$$
g w\left(1-w^{2}\right)^{1 /(\gamma-1)}=e^{a(\xi)}
$$

and

$$
\frac{w^{2}}{1-w^{2}} \frac{\partial}{\partial \xi} \ln g w=b(\xi)
$$

where $a(\xi)$ and $b(\xi)$ are arbitrary functions possessing derivatives of fourth and third order respectively. Our problem is to find what functions $w(\xi, \eta)$ and $g(\xi, \eta)$ are consistent with (3), (4) and (5).

Elimination of $g w$ between (4) and (5) and of $g$ between (3) and (4) results in two partial differential equations restricting $w$ :

$$
\frac{\partial w^{2}}{\partial \xi}=m\left(w^{2}, \xi\right)
$$

and

$$
\frac{\partial^{2} w^{2}}{\partial \xi^{2}}+\frac{\partial^{2} w^{2}}{\partial \eta^{2}}=f\left(w^{2}\right)\left[\left(\frac{\partial w^{2}}{\partial \xi}\right)^{2}+\left(\frac{\partial w^{2}}{\partial \eta}\right)^{2}\right]+h\left(w^{2}\right) a^{\prime \prime}(\xi)
$$

where

$$
\begin{aligned}
m\left(w^{2}, \xi\right) & \equiv \frac{1-w^{2}}{\beta w^{2}}\left\{b(\xi)-\left\lfloor b(\xi)+a^{\prime}(\xi)\right] w^{2}\right\}, \\
f\left(w^{2}\right) & \equiv \frac{(2 \beta+1) w^{4}-2 w^{2}+1}{w^{2}\left(1-w^{2}\right)\left[1-(2 \beta+1) w^{2}\right]}, \\
h\left(w^{2}\right) & \equiv \frac{2 w^{2}\left(1-w^{2}\right)}{1-(2 \beta+1) w^{2}},
\end{aligned}
$$

and

$$
\beta \equiv 1 /(\gamma-1)
$$


Use of (6) to eliminate $\partial w^{2} / \partial \xi$ and $\partial^{2} w^{2} / \partial \xi^{2}$ from (7) yields:

$$
\frac{\partial^{2} w^{2}}{\partial \eta^{2}}=f\left[m^{2}+\left(\frac{\partial w^{2}}{\partial \eta}\right)^{2}\right]+h a^{\prime \prime}-m \frac{\partial m}{\partial w^{2}}-\frac{\partial m}{\partial \xi} .
$$

Evaluation of $\partial^{3} w^{2} / \partial \xi \partial \eta^{2}$ from (8) and $\partial^{3} w^{2} / \partial \eta^{2} \partial \xi$ from (6) leads to the requirement:

$$
\text { (9) }\left(\frac{\partial w^{2}}{\partial \eta}\right)^{2}=\frac{\begin{array}{c}
m^{2} \partial^{2} m / \partial w^{22}+2 m \partial^{2} m / \partial \xi \partial w^{2}+\partial^{2} m / \partial \xi^{2}-f m(2 \partial m / \partial \xi \\
\left.+m \partial m / \partial w^{2}\right)-f^{\prime} m^{3}-m h^{\prime} a^{\prime \prime}-h a^{\prime \prime \prime}+h(\partial m / \partial w) a^{\prime \prime}
\end{array}}{-\partial^{2} m / \partial w^{22}+f \partial m / \partial w^{2}+f^{\prime} m} \text {. }
$$

Case I: $m \neq 0$. If $m \neq 0$, (9) determines $\partial w^{2} / \partial \eta$ as a function of $w^{2}$ and $\xi$, for brevity

$$
\frac{\partial w^{2}}{\partial \eta}=l\left(w^{2}, \xi\right)
$$

Evaluation of $\partial^{2} w^{2} / \partial \xi \partial \eta$ from (10) and $\partial^{2} w^{2} / \partial \eta \partial \xi$ from (6) then leads to the relation

$$
\frac{\partial m}{\partial w^{2}} l-m \frac{\partial l}{\partial w^{2}}-\frac{\partial l}{\partial \xi}=0
$$

from which it follows that

$$
w=w(\xi),
$$

where $w(\xi)$ is arbitrary by virtue of the functions $a(\xi)$ and $b(\xi)$. (11) and (4) imply

$$
g=g(\xi)
$$

and (3) then requires

$$
\ln g=\mathcal{A} \xi+B \text {. }
$$

This restriction of $g$ limits the streamlines to concentric circles or parallel straight lines.

Case II: $m=0$. If $m=0$, it is implied (6a) that $b(\xi)=a^{\prime}(\xi)=0$ and from (6)

$$
w=w(\eta) .
$$

Letting $a(\xi)$ be an arbitrary constant, (4) implies

$$
g=g(\eta)
$$

and (3) then requires 


$$
\ln g=\Phi \eta+\varepsilon \text {. }
$$

This restricts the streamlines to a radial pencil of straight lines or parallel straight lines.

The function $w(\eta)$ is not arbitrary, but is given implicitly by (12) and (4). By virtue of $b(\xi)$ being zero, the $\bar{w}$ field is here irrotational.

This same geometric restriction applies also to the singular, physically trivial, case when $w^{2}=1$. This is easily shown by use of (1a).

We have now established the theorem:

The only isothermal streamline patterns possible in steady plane flow of an ideal gas without body forces consist of concentric circles, radial straight lines, and parallel straight lines. ${ }^{2}$

This theorem was established from purely local considerations and, hence, remains valid for the separate regions of flow fields containing shocks or other discontinuities. The proof given presupposes the existence of (piecewise) continuous third order derivatives of $w$. It is of interest to note that the class of irrotational gas flows includes exactly the same flow patterns of the type considered as does the more general rotational class.

\section{REFERENCES}

1. M. Munk and R. Prim, On the canonical form of the equations of steady motion of a perfect gas, Naval Ordnance Laboratory Memorandum No. 9169, June, 1947.

2. P. Nemenyi and R. Prim, On the equations of plane rotational flow of a perfect gas in natural coordinates and on plane rotational gas flow with constant velocity or constant vorticity along the streamlines, Naval Ordnance Laboratory Memorandum No. 9264, August, 1947.

3. D. Gilbarg, Flow patterns common to certain classes of plane fluid motions, Journal of Mathematics and Physics, Massachusetts Institute of Technology vol. 26 (1947).

Naval ORDNANCE Laboratory

${ }^{2}$ It has been called to the author's attention that D. Gilbarg has independently established this theorem for the special case of irrotational flow [3]. 